Research Paper

\title{
Multifunctional Gold Nanostar Conjugates for Tumor Imaging and Combined Photothermal and Chemo-therapy
}

\author{
Haiyan Chen ${ }^{1 *}$, Xin Zhang $1^{*}$, Shuhang Dai ${ }^{3}$, Yuxiang Ma ${ }^{1}$, Sisi Cui ${ }^{1}$, Samuel Achilefu ${ }^{2 \bowtie}$, Yueqing Gu ${ }^{\circledR}$ \\ 1. Department of Biomedical Engineering, State Key Laboratory of Natural Medicines and School of Life Science and Technology, China \\ Pharmaceutical University, 24 Tongiia Lane, Gulou District, Nanjing 210009, China \\ 2. Department of Radiology, School of Medicine, Washington University, St. Louis, Missouri, United States \\ 3. Department of Pharmaceutical Science, School of Pharmacy, China Pharmaceutical University, 24 Tongjia Lane, Gulou District, Nanjing \\ 210009, China \\ ${ }^{*}$ Haiyan Chen and Xin Zhang contributed equally to the work.
}

Corresponding author: Samuel Achilefu, PhD. Washington University in St. Louis, Phone: +1-314-362-8599 Email: achilefus@mir.wustl.edu Or Yueqing Gu, PhD. China Pharmaceutical University. Phone: +86-25-83208205 Email: guyueqing@cpu.edu.cn.

( ) Ivyspring International Publisher. This is an open-access article distributed under the terms of the Creative Commons License (http://creativecommons.org/ licenses/by-nc-nd/3.0/). Reproduction is permitted for personal, noncommercial use, provided that the article is in whole, unmodified, and properly cited.

Received: 2013.05.06; Accepted: 2013.06.28; Published: 2013.08.03

\begin{abstract}
Uniform gold nanostars (Au NS) were conjugated with cyclic RGD (cRGD) and near infrared (NIR) fluorescence probe (MPA) or anti-cancer drug (DOX) to obtain multi-functional nanoconstructs, Au-cRGD-MPA and Au-cRGD-DOX respectively. The NIR contrast agent Au-cRGD-MPA was shown to have low cytotoxicity. Using tumor cells and tumor bearing mice, these imaging nanoparticles demonstrated favorable tumor-targeting capability mediated by RGD peptide binding to its over-expressed receptor on the tumor cells. The multi-therapeutic analogue, Au-cRGD-DOX, integrates targeting tumor, chemotherapy and photo-thermotherapy into a single system. The synergistic effect of photo-thermal therapy and chemotherapy was demonstrated in different tumor cell lines and in vivo using SI80 tumor-bearing mouse models. The viability of MDA-MB-23I cells was only $40 \%$ after incubation with Au-cRGD-DOX and irradiation with NIR light. Both tail vein and intratumoral injections showed Au-cRGD-DOX treated mice exhibiting the slowest tumor increase. These results indicate that the multifunctional nanoconstruct is a promising combined therapeutic agent for tumor-targeting treatment, with the potential to enhance the anti-cancer treatment outcomes.
\end{abstract}

Key words: gold nanostar, tumor-targeting, RGD receptor, near infrared imaging, photo-thermal therapy.

\section{Introduction}

Although enormous efforts have been expended into the development of effective methods for tumor therapy in the past decades, cancer remains one of the most difficult diseases to treat as well as a leading cause of morbidity and mortality. The limitations of the existing single-modality treatments such as chemotherapy, radiotherapy, immunotherapy, gene therapy and thermotherapy are well known, necessitating the use of multi-modality treatments to improve treatment outcomes in recent years $[1,2]$. In contrast to the single treatment regimen, combination therapies such as chemotherapy and thermotherapy have been reported to synergistically improve the antitumor efficacy with reduced side effects $[3,4]$. 
Gold nanoconstructs have been intensely investigated as the platform for multi-modality treatment, especially for the chemo-photothermal therapy because of two major features. First, the surface of gold nanomaterials can be easily functionalized with chemotherapeutics and other functional ligands through the reactive groups of the capping agents (eg. amino group, and carboxyl group) or through thiol-mediated binding to gold surface. Second, when the tunable surface plasmon resonance (SPR) of gold nanoconstructs matches the wavelength of irradiating light, coherent oscillations of electrons in the conduction band will induce photothermal conversion by converting the absorbed light to heat [5]. More importantly, unlike the visible light, light at near-infrared (NIR) light is poorly absorbed by endogenous chromophores in tissues, including water, melanin and hemoglobin. This favors deep penetration of NIR light into tissue, thus excitation of the non-superficial target objects in the body [6].

A variety of gold nanoconstructs, such as gold naonrods [7, 8], nanoshells [9], nanocages [10], hollow nanospheres [11], and branched gold nanoparticles [12] have been proved to exhibit plasmon resonance in the NIR region, and produce hyperthermia upon the NIR laser irradiation. Huang et al. [13] proposed the concept of plasmon photothermal therapy (PPTT) using gold nanoparticles for localized photothermal therapy. Particularly, interest in the use of branched gold nanoparticles or gold nanostars (Au NS) with the plasmon peaks tunable in the infrared range has increased because of the ease of synthesis for large scale production, high surface-to-volume ratio useful for improving drug loading efficiency, and surface plasmon resonance in the "NIR transparent window" for deep tissue imaging [14-17]. Yuan et al. [18] reported that gold nanostars functionalized with TAT-peptides efficiently internalized in cells and demonstrated that these particles are promising agents for cancer therapy via photothermolysis.

In order to increase the accumulation of nanoparticles in tumor sites and lower the side effects on normal tissues, many investigators have functionalized nanoparticles with ligands such as aptamer, peptide, and oligosaccharide for active tumor targeting. Among the various tumor-targeted ligands, arginine-glycine-aspartic acid (RGD) peptide is one of the most effective and widely used compounds for delivery imaging agents and drugs to tumors [19]. The RGD receptor, integrin $\alpha_{v} \beta_{3}$, plays a critical role in regulating tumor growth and metastasis as well as tumor-mediated angiogenesis. It is over-expressed on the surface of a variety of tumor cells including human malignant melanoma, breast cancer, and ad- vanced glioblastoma [20]. To improve the affinity between the ligands and $a_{v} \beta_{3}$ receptor, diverse multivalent cyclic RGD-based peptides have been designed. Choi et al. [21] demonstrated that a dye-RGD peptide conjugate provides high signal-to-background ratio when targeted to cancer cells. Similarly, Zhou et al. [22] also demonstrated 99mTc-labeled cyclic RGD dimmer efficiently detected integrin $\alpha_{v} \beta_{3}$-positive tumors.

In the present study, Au NS was prepared by reducing $\mathrm{Au}^{3+}$ in HEPES buffer using the "green" method reported by Xie et al. [23] Doxorubicin (DOX) and cyclic RGD (cRGD) peptide was covalently linked to the surface of $\mathrm{Au}$ NS via glutathione (GSH) to form Au-DOX, Au-cRGD, and Au-cRGD-DOX. We also labeled the cRGD-modified Au NS (Au-cRGD) with a hydrophilic indocyanine green (ICG) derivative (MPA) to investigate the biodistribution of Au NS and to assess the selective uptake of Au NS in tumors after attachment of cRGD. The affinity of Au-cRGD-DOX for different tumor cells was examined by fluorescence microscopy and the selective tumor-uptake of Au-cRGD-MPA was verified by monitoring its in vivo dynamics in real time. Furthermore, we also compared the chemo-photothermal therapeutic efficacy of Au-cRGD-DOX with free DOX, Au NS, and Au-DOX in cells and tumor-bearing mice by irradiation with NIR laser. Finally, we investigated the influence of administrative routes on anti-tumor effect of Au-cRGD-DOX by injecting DOX, Au NS, Au-DOX, Au-cRGD-DOX intratumorally and intravenously.

\section{Materials and Methods}

\section{Materials}

4-(2-hydroxyethyl)-1-piperazineethane-sulfonic acid (HEPES) was obtained from Sigma Aldrich (Tianjing, China). $\mathrm{HAuCl}_{4}$ was purchased from Guoyao Reagent Corporation (Shanghai, China) and used as received. Reduced glutathione (GSH) was obtained from Sangon Biotech Co., LTD (Shanghai, China) and cyclic RGD peptide (cyclo (Arg-Gly-Asp-DTyr-Lys)) was purchased from GL Biochem Co., Ltd (Shanghai, China). MPA (MW: 995) was prepared in our laboratory. Doxorubicin (DOX, MW: 149.15), 1-ethyl-3-[3-diMethylaminopropyl] carbodiimide hydrochloride (EDC), N, $\mathrm{N}^{\prime}$-Dicyclohexylcarbodiimide

(DCC), N-hydroxysuccinimide (NHS), 3-(4, 5- diMethylthialzol-2-yl)-2, 5-diphe-nyltetrazolium bromide (MTT), RPMI 1640, fetal bovine serum (FBS), penicillin, streptomycin, and trypsin-EDTA were purchased from commercial sources. Ultrapure millipore water (18.2 MU) was used. All other solvents and reagents 
used in this study were certified analytical reagent grade.

\section{Preparation of Au NS and its conjugates}

\section{Synthesis of Au NS and Au-Glutathione (Au-GSH)}

All the glassware should be washed by Aqua Regia and rinsed by ultrapure water repeatedly. In a typical experiment, aqueous $\mathrm{HAuCl}_{4}$ solution $(10 \mu \mathrm{L}$, $40 \mathrm{mM})$ was added to HEPES solution $(2.0 \mathrm{~mL}, 140$ $\mathrm{mM}, \mathrm{pH}=7.4 \pm 0.1)$ at $25^{\circ} \mathrm{C}$, and the reaction solution was degassed by $\mathrm{N}_{2}$ purging for $10 \mathrm{~min}$. Without shaking, Au NS formed in 1 hour, and the color of the solution changed from colorless to dark green. GSH (5.0 mg) was added to the solution after the synthesis finished and the mixture was left overnight to form the nanoconstruct (Au-GSH). After $12 \mathrm{~h}$, the solution was centrifuged at $8000 \mathrm{rpm}$ for $15 \mathrm{~min}$. The supernatant was discarded, and ultrapure water $(1.0 \mathrm{ml})$ was added to re-dissolve the precipitate.

\section{Synthesis of Au-cRGD, Au-DOX and Au-cRGD-Doxorubicin (Au-cRGD-DOX)}

Cyclic RGD peptide was immobilized onto Au-GSH by using coupling agents (EDC and NHS) [24]. Briefly, EDC (5.0 mg) and NHS (4.6 mg) were added into Au-GSH solution $(5.0 \mathrm{ml})$ prepared above and the mixture was stirred at room temperature for 4 h. After the reaction, cRGD (3.1 mg) and DOX (5.4 mg) were added to the activated Au-GSH solution simultaneously and the new mixture was incubated overnight. Subsequently, the solution was centrifuged at $8000 \mathrm{rpm}$ for $15 \mathrm{~min}$. The residual small molecules including EDC, NHS and RGD in the supernatant were discarded. $2.0 \mathrm{~mL}$ ultrapure water was utilized to dissolve the precipitate. Au-cRGD and Au-DOX were synthesized according to the above method except DOX or cRGD was not included in the reaction.

\section{Synthesis of Au-cRGD-MPA conjugates}

MPA (1.0 mg) was activated with DCC and NHS (molar ratio of MPA: DCC: NHS is 1:1.2: 1.5) at room temperature in the dark for $3 \mathrm{~h}$. The above activated MPA $(200 \mu \mathrm{L})$ was then uniformly mixed with purified Au-cRGD $(1.0 \mathrm{~mL})$ and incubated at room temperature with stirring in the dark for $12 \mathrm{~h}$. The incubation product was purified by centrifugation at 8000 rpm for $15 \mathrm{~min}$ to remove the small molecules in the supernatant. Ultrapure water $(0.5 \mathrm{~mL})$ was used to re-dissolve the precipitate.

\section{Characterization}

Morphology and Size Distribution

The size and morphology of Au NS and
Au-cRGD-DOX were measured by JEOL JEM-2100 and transmission electron microscope (TEM). A dilute solution of the sample was added dropwise into 400-mesh carbon-coated copper grids and the excessive solvent was immediately evaporated before microscopy. The hydrodynamic size and polydispersity of $\mathrm{Au}$ NS and Au-cRGD-DOX were measured by Mastersizer 2000 Laser Particle Size Analyzer (Malvern, British) at $25^{\circ} \mathrm{C}$. A helium-neon laser $(10 \mathrm{~mW}$ max, Wand wavelength of $633 \mathrm{~nm}$ ) was used as the light source. Polydispersity values were utilized to evaluate the distribution of the nanoparticles population.

\section{Optical Properties of Au NS and its Conjugates}

The absorption spectra of Au NS, MPA, Au-MPA, Au-cRGD-MPA, DOX, Au-DOX and Au-cRGD-DOX were acquired with Lambda 25 UV-Vis spectrophotometer (Perkin Elmer, US). The corresponding fluorescence spectra of the materials were measured at room temperature using a S2000 eight-channel optical fiber spectrofluorometer equipped with a Xenon lamp.

Photo-thermal effect of the nano-conjugates of Au NS in solutions

Change in temperature as a function of time was used to determine the photothermal effect of the nanoparticles. The DMEM medium with PBS, Au-MPA, Au-cRGD-MPA, Au-DOX and Au-cRGD-DOX (50 $\mu \mathrm{g} / \mathrm{mL}$ ) were each added into different wells of the 96-well plate.The temperature increase was recorded after exposing the samples to a $765 \mathrm{~nm}$ laser (Shanghai nLight Optics Company) at different light irradiation time ( $0 \mathrm{~min}, 1 \mathrm{~min}, 5 \mathrm{~min}$ ). The beam diameter was $1 \mathrm{~cm}$ and the power density of laser source was fixed at $1.0 \mathrm{~W} / \mathrm{cm}^{2}$.

Drug loading efficiency and in vitro drug release profile

To calculate the DOX loading efficiency, Au-cRGD-DOX was collected by centrifugation and the unconjugated DOX in the supernatant was quantified by using DOX UV calibration curve at $481 \mathrm{~nm}$. The DOX loading was calculated according to the formula: drug loading (DL) $=$ (mass of drug conjugated with $\mathrm{Au}$ NS / total mass of drug conjugated and $\mathrm{Au}$ NS).

To investigate the in vitro DOX release profile, PBS containing $10 \%$ fetal bovine serum with or without GSH $(10 \mathrm{mM})$ were used to dissolve $\mathrm{Au}-\mathrm{cRGD}-\mathrm{DOX}$ in a dialysis bag. Dislysate $(2 \mathrm{~mL})$ was withdrawn at different time intervals $(0.5 \mathrm{~h}, 1 \mathrm{~h}, 2 \mathrm{~h}, 6$ $\mathrm{h}, 10 \mathrm{~h}, 16 \mathrm{~h}, 20 \mathrm{~h}$ and $24 \mathrm{~h}$ ) and an equal volume of PBS was added to the solution outside of the dialysis 
bag to maintain the net volume of the system. The absorbance peak $(481 \mathrm{~nm})$ of the solutions were detected and the quantities of the free dugs were calculated from the standard curve defined by the equation $y=0.3246 x-0.0042\left(R^{2}=0.9996\right)$.

\section{Cell studies}

\section{Cell culture}

The human cell lines, MDA-MB-231 (breast cancer), Bel-7402 (liver cancer) and MCF-7 (breast cancer) used in this study, were all purchased from American Type Culture Collection (ATCC, USA). The cell lines were cultured in RPMI-1640 medium supplemented with $10 \%(\mathrm{v} / \mathrm{v})$ calf serum, penicillin $\left(100 \mathrm{U} \mathrm{ml}^{-1}\right)$, and streptomycin $\left(100 \mathrm{mg} \mathrm{ml}^{-1}\right)$. The cells were maintained at $37{ }^{\circ} \mathrm{C}$ in a humidified atmosphere containing $5 \%$ $\mathrm{CO}_{2}$.

\section{Assessment of cell uptake}

The targeting ability of DOX, Au-DOX and $\mathrm{Au}-\mathrm{cRGD}-\mathrm{DOX}$ were evaluated using the three tumor cells lines, MDA-MB-231, Bel7402, and MCF-7 cells. The cells were seeded in laser confocal fluorescence microscope (LCFM) culture dishes with a density of $4 \times 10^{5}$ cells/well and subsequently incubated at $37^{\circ} \mathrm{C}$ in a humidified atmosphere containing $5 \% \mathrm{CO}_{2}$. When the whole cells reached $70 \% \sim 80 \%$ confluence, $20 \mu \mathrm{L}$ of DOX, Au-DOX and Au-cRGD-DOX were added into different dishes and then incubated for $1 \mathrm{~h}$. In addition, MDA-MB-231 cells were treated with Au-cRGD-DOX for $8 \mathrm{~h}$, followed by incubation with Hoechst 33342 solutions $(100 \mu \mathrm{L}, 10 \mu \mathrm{g} / \mathrm{mL})$ for 30 $\mathrm{min}$. The information was used to determine the basis for understanding the intracellular kinetics of Au-cRGD-DOX. Subsequently, the cells were washed three times with Dulbecco's phosphate buffered saline (PBS, pH 7.0) before examining the probe's affinity for cancer cells by LCFM (FV1000, Olympus, Japan). The red fluorescence of DOX was captured under $488 \mathrm{~nm}$ light excitation. Intracellular uptake of the different probes was determined from the red fluorescence intensity in the region of interest (ROI) by using Scion Image software.

\section{Cell apoptosis evaluation}

To evaluate the therapeutic efficacy of Au NS, DOX, Au NS (NIR light), Au-DOX (NIR light) and Au-cRGD-DOX (NIR light), the induction of apoptosis in MDA-MB-231 cells was studied by determining the cell number and cell morphology. Specifically, cells were plated at a density of $4 \times 10^{5}$ cells/dish in confocal specific dish and subsequently incubated under the culture condition for $36 \mathrm{~h}$. The cells were treated with $100 \mu \mathrm{L}$ solutions of Au NS, DOX $(5.0 \mu \mathrm{g} / \mathrm{mL})$,
Au-DOX and Au-cRGD-DOX (conjugated DOX: 5.0 $\mu \mathrm{g} / \mathrm{mL}$ ) for $4 \mathrm{~h}$. The photothermal treatment was monitored using a $765 \mathrm{~nm}$ high power multimode pump laser (Ennai, Shanghai, China). The beam diameter was $2.0 \mathrm{~cm}$ and the power density of laser source was fixed at $1.0 \mathrm{~W} / \mathrm{cm}^{2}$. The cells were exposed under the $765 \mathrm{~nm}$ laser for $10 \mathrm{~min}$ and then washed three times with PBS $(\mathrm{pH}=7.0)$. The residual cells and the morphological changes were displayed by the differential interference contrast (DIC) mode of LCFM. For MDA-MB-231 and MCF-7 cells, the induced cell apoptosis was also characterized by cell membrane and cell nucleus staining. Annexin V-FITC $(5 \mu \mathrm{L})$ and propidium iodide $(5 \mu \mathrm{L})$ were added into the culture dish and incubated at room temperature in the dark for $10 \mathrm{~min}$. After adding $500 \mu \mathrm{L}$ of binding buffer, the cells were directly visualized by LCFM (40 $x$ objective magnification). The red and green fluorescence intensity was identified from the ROI of each image by Scion Image software.

To quantitatively evaluate the effect of the nanomaterials on MDA-MB-231 cells, MTT assay was also carried on Au NS, DOX, Au-DOX and Au-cRGD-DOX using a concentration range of 0.7 $\mu \mathrm{g} / \mathrm{mL}$ to $446.4 \mu \mathrm{g} / \mathrm{mL}$ for Au NS and its conjugates and $0.02 \mu \mathrm{g} / \mathrm{mL}$ to $12.5 \mu \mathrm{g} / \mathrm{mL}$ for free DOX and conjugated DOX. After the cells were incubated for 4 $\mathrm{h}$, the photothermal treatment was then performed using a $765 \mathrm{~nm}$ laser. The beam diameter was $2.0 \mathrm{~cm}$ and the power density of laser source was fixed at 1.0 $\mathrm{W} / \mathrm{cm}^{2}$. The cells were exposed under the $765 \mathrm{~nm}$ laser for $15 \mathrm{~min}$, and then incubated for another $12 \mathrm{~h}$. Each well was replaced and the cells were washed three times with PBS ( $\mathrm{pH}$ 7.0) before addition of $20 \mu \mathrm{L}$ of MTT solution $(5.0 \mu \mathrm{g} / \mathrm{mL})$. After incubating another $4 \mathrm{~h}$, the medium containing MTT was carefully removed from each well and DMSO $(150 \mu \mathrm{L})$ was added to each well to dissolve the purple crystals. The plates were gently shaken for $10 \mathrm{~min}$ at room temperature before measuring the absorbance at $580 \mathrm{~nm}$. All test samples were assayed in quadruplicate and the cell viability was calculated using the following formula: Cell viability $=($ Mean absorbance of test wells - Mean absorbance of medium control wells) / (Mean absorbance of untreated wells - Mean absorbance of medium control well) $\times 100 \%$.

\section{Animal experiments}

\section{Animal subjects and tumor model}

Athymic nude mice and normal (Kunming) mice were purchased from Charles River Laboratories (Shanghai, China) for in vivo investigation. All animal experiments were carried out in compliance with the 
Animal Management Rules of the Ministry of Health of the People's Republic of China (Document NO. 55, 2001) and the guidelines for the Care and Use of Laboratory Animals of China Pharmaceutical University.

\section{Dynamic distribution of Au-cRGD-MPA in tumor bearing mice}

To investigate the targeting ability of the probes, two types of tumor models were established by subcutaneous injection of MDA-MB-231 (high av $\beta 3$ receptor expression) and MCF-7 (low $\mathrm{a}_{\mathrm{v}} \beta_{3}$ receptor expression) tumor cells ( $5 \times 10^{6}$ in $80 \mu \mathrm{L}$ of PBS) into the axillary fossa of female athymic nude mice $(n=5$ for each model).

As the tumors grew to a diameter of up to $0.5 \mathrm{~cm}$, Au-cRGD-MPA $(0.2 \mathrm{~mL}, 10 \mathrm{mg} / \mathrm{kg})$ was injected into MDA-MB-231 and MCF-7 tumor-bearing mice through the vena caudalis. The nude mice were then immobilized on a Lucite jig. NIR imaging system equipped with a $765 \mathrm{~nm}$ laser as excitation source was employed to trace the distribution of the probe. The background image of the mice was taken prior to the injection. A series of images were collected at $5 \mathrm{~min}, 1$ $\mathrm{h}, 2 \mathrm{~h}, 4 \mathrm{~h}, 12 \mathrm{~h}, 24 \mathrm{~h}$ and $48 \mathrm{~h}$ post-injection respectively. To further compare the targeting ability of the probe for MDA-MB-231 and MCF-7 tumors, tumor to normal tissue contrast ratios $(\mathrm{T} / \mathrm{N})$ at different time points were calculated by using ROI functions of Scion Image software. In each case, the background fluorescence that was measured before injection of the conjugates was subtracted from the post injection fluorescence.

In order to confirm the bio-distribution of the Au-cRGD-MPA in living mice, some major organs (heart, liver, spleen, lung, kidney, intestine and tumor of the subject MDA-MB-231 tumor-bearing mice were excised at 48 post-injection and detected by NIR fluorescence imaging system.

\section{Therapeutic efficacy evaluation of Au-cRGD-DOX}

S180 tumor model for therapy evaluation was established by subcutaneously inoculating S180 cells $\left(\sim 8 \times 10^{6}\right)$ into the nether axillary fossa in the mice $(\mathrm{n}=$ $60)$. The mice were investigated after 7 days of inoculating.

When the tumor was fully developed, the mice were randomly divided into six groups $(n=10)$ for each group. We intratumoral injected $50 \mu \mathrm{L}$ of Saline, $1.4 \mathrm{mg} / \mathrm{kg}$ DOX, $50 \mathrm{mg} / \mathrm{kg}$ Au NS, $50 \mathrm{mg} / \mathrm{kg} \mathrm{Au}-\mathrm{DOX}$ (containing $3.2 \mathrm{mg} / \mathrm{kg} \quad \mathrm{DOX}$ ) or $50 \mathrm{mg} / \mathrm{kg}$ Au-cRGD-DOX (containing $1.4 \mathrm{mg} / \mathrm{kg}$ DOX) into the mice and Au-cRGD-DOX was also intravenously administered in the mice ( $50 \mu \mathrm{L}, 75 \mathrm{mg} / \mathrm{kg}$ ). Groups 3, 4, 5 and 6 were exposed to $1.0 \mathrm{~W} / \mathrm{cm}^{2} \mathrm{NIR}$ light for $10 \mathrm{~min}$ at $8 \mathrm{~h}$ after intravenous injection of the nanoparticles. Subsequently, each mouse underwent 4 intratumoral injection or tail vein injections once every other day. The tumor volume and body weight of each mouse were monitored every three days for 26 days. The tumor volume was calculated as length $\times$ (width) ${ }^{2}$ $\times 1 / 2$ with a caliper. The survival rate of the treated mice was calculated according to the equation: Survival rate $=\mathrm{Ns} / \mathrm{Nt} \times 100 \%$, where $\mathrm{Ns}$ and $\mathrm{Nt}$ represent the number of surviving mice and the number of total mice in each group, respectively.

To monitor the photothermal effect of laser exposure, intratumoral injection of PBS, Au NS, Au-DOX and Au-cRGD-DOX (50 $\mu \mathrm{g} / \mathrm{mL})$ separately into S180 tumor-bearing mice was followed by irradiation with a $765 \mathrm{~nm}$ laser (beam diameter: $1 \mathrm{~cm}$, powder density: $1.0 \mathrm{~W} / \mathrm{cm}^{2}$.) for $10 \mathrm{~min}$. A needle temperature probe (NR81530, TES, Shenzhen Yuanhengtong technology company) was used to detect the intratumoral temperature of different groups after laser treatment.

\section{Ex vivo Histopathology}

To further investigate the therapeutic effects of Au-cRGD-DOX on S180 tumor-bearing mice treated by tail vein or intratumoral injection, the tumors were excised for histopathologic analysis on day 16 post-injection. The tissue was fixed with $10 \%$ neutral buffered formalin and embedded in paraffin. The sliced tumors $(8 \mathrm{~mm})$ were stained with Hematoxylin and Eosin (H\&E) and observed by a BX41 bright field microscopy (Olympus).

\section{Statically Analysis}

Significant differences were determined using the Student's t-test where differences were considered significant $(p<0.05)$. All data are expressed as mean \pm standard error of the mean.

\section{Result and Discussion}

\section{Preparation and characterization of Au NS and its nanoconjugates}

Bio-compatible $\mathrm{Au}$ NS was prepared by reducing $\mathrm{HAuCl}_{4}$ with HEPES, a popular $\mathrm{pH}$ buffer used extensively in tissue culturing [18, 25]. The process of functionalization is illustrated in Figure S1 (Supplementary Material). The morphology, size, and polydispersity index of $\mathrm{Au}$ NS and Au-cRGD-DOX were measured by TEM and Laser Particle Size Analyzer. TEM analysis showed average size of $25 \mathrm{~nm}$ and $80 \mathrm{~nm}$ for $\mathrm{Au}$ NS and Au-cRGD-DOX, respectively (Fig. 1A and Fig. 1B). The hydrodynamic di- 
ameters of $\mathrm{Au}$ NS and Au-cRGD-DOX were $32.6 \mathrm{~nm}$ (polydispersity index: 0.216 ) and $58.4 \mathrm{~nm}$ (polydispersity index: 0.232), respectively (Fig. 1C and Fig. 1D). It was clear that upon the nanostars functionalization with cRGD and DOX, the nanostars aggregated into larger uniform clusters. In addition, the DOX loading rate was $2.8 \%(\mathrm{w} / \mathrm{w})$ by quantifying the unconjugated DOX in the supernatant.

The modification of Au NS by DOX, RGD and MPA was monitored by UV-Vis absorption spectra and fluorescence spectra (Fig. 2). Consistent with the previous reports $[18,26]$, Au NS showed distinct absorption peak at $740 \mathrm{~nm}$ (Fig. 2A and Fig. 2C). The absorption peak at $350 \mathrm{~nm}$ could be attributed to the molecules on the surface of $\mathrm{Au}$ NS. The conjugates, Au-MPA and Au-cRGD-MPA, still exhibited the characteristic absorption peaks of MPA $(780 \mathrm{~nm})$. However, the characteristic absorption peak of Au NS became indistinct after conjugation with MPA, cRGD or DOX, which was in agreement with the TEM and DLS results. Although the absorption spectrum of $\mathrm{Au}$ NS (650-780 nm) and the emission spectrum of MPA
(750-810 nm) partly overlapped each other in NIR region, the fluorescence of MPA was maintained after conjugation with $\mathrm{Au} \mathrm{NS}$, facilitating the use of Au-cRGD-MPA for in vivo imaging (Fig. 2A and Fig. 2B). The DOX loading of $2.8 \%(\mathrm{w} / \mathrm{w})$ was determined by quantifying the unconjugated DOX in the supernatant. Additionally, Fig. 2D suggests that the fluorescence peak emitted by DOX did not shift after conjugation with $\mathrm{Au}$ NS. To further investigate the stability of the nanoparticles, the UV-Vis spectra of $\mathrm{Au}$ NS and Au-cRGD-DOX in fetal bovine serum immediately and after $8 \mathrm{~h}$ incubation were measured (Fig. 2E). The result indicates that there was no obvious change in the absorption spectra. The in vitro DOX release profile is shown in Fig. 2F. Obviously, DOX release was much faster in PBS containing $10 \%$ fetal bovine serum and GSH $(10 \mathrm{mM})$ than in the incubation system without GSH. Almost $80 \%$ of DOX was released from Au-cRGD-DOX in the initial $10 \mathrm{~h}$ when incubated with GSH, and a complete release was achieved at $24 \mathrm{~h}$ incubation. In contrast, only $30 \%$ of the drug was released in the solution without GSH.
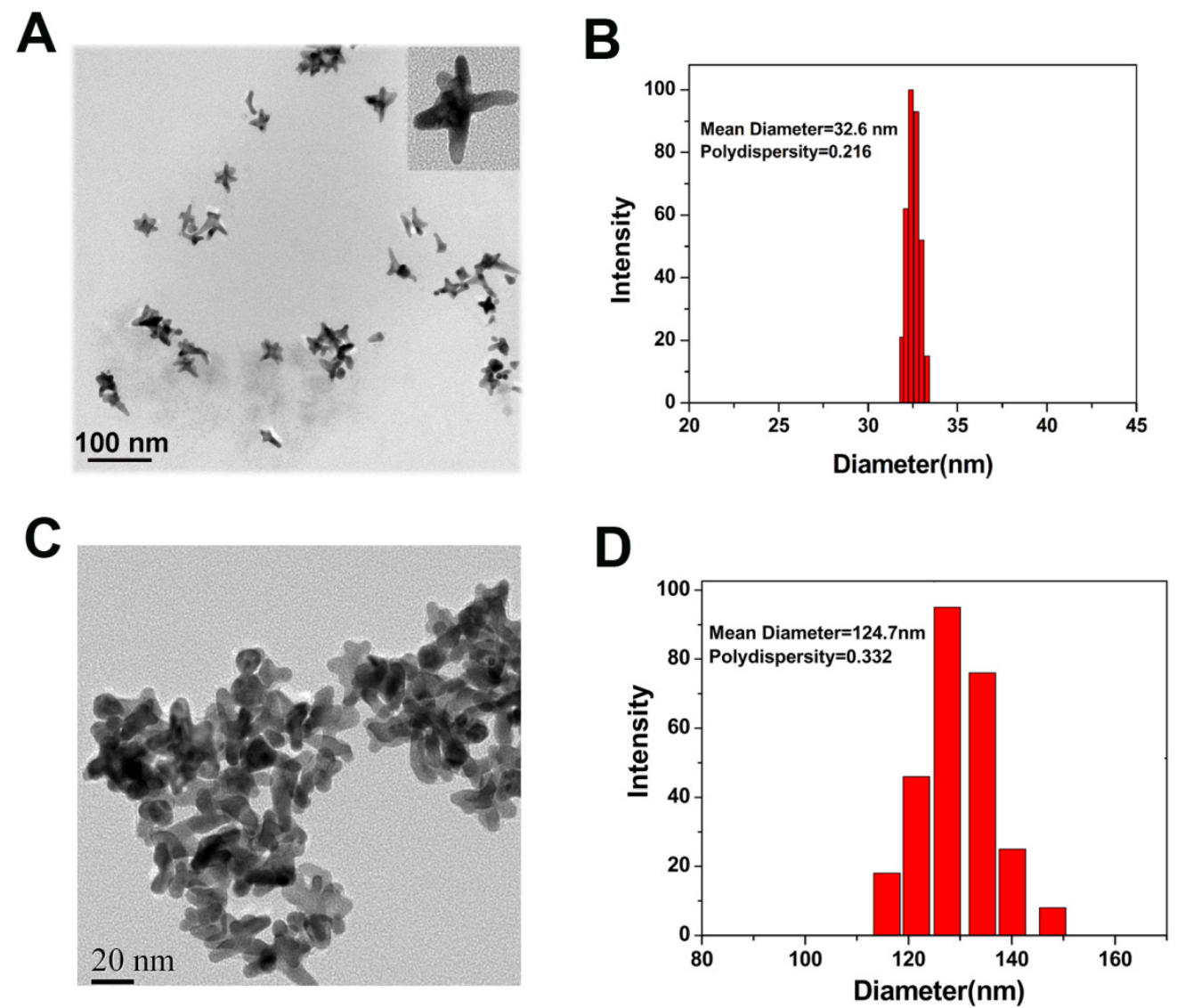

Fig I. TEM images of Au Ns A) before (as-synthesized) and B) after conjugation with cRGD and DOX; Hydrodynamic diameter of Au Ns C) before (as-synthesized) and D) after conjugation with cRGD and DOX measured by laser particle size analyzer. 
A

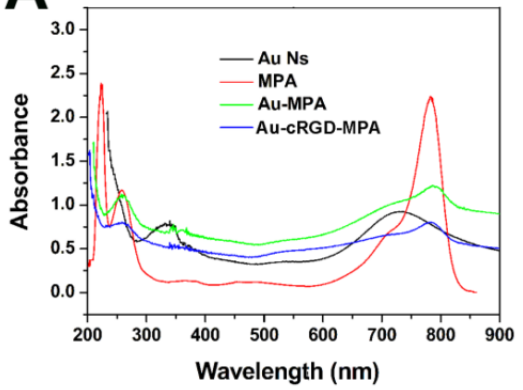

C

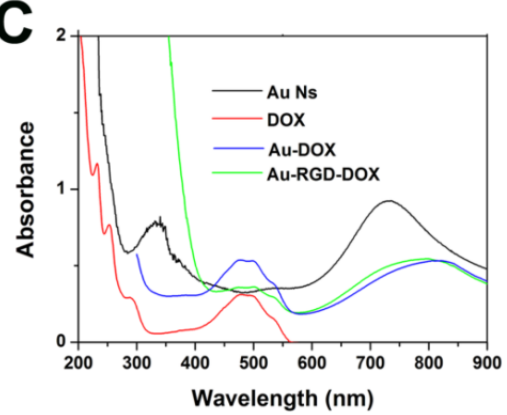

E

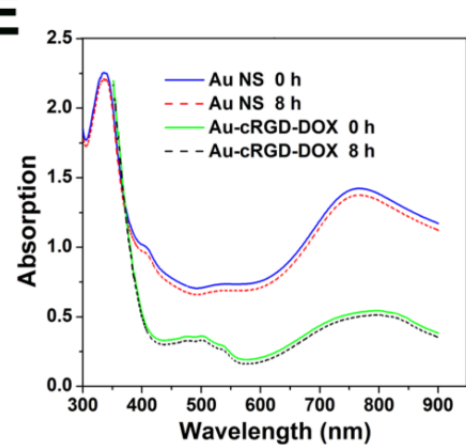

B

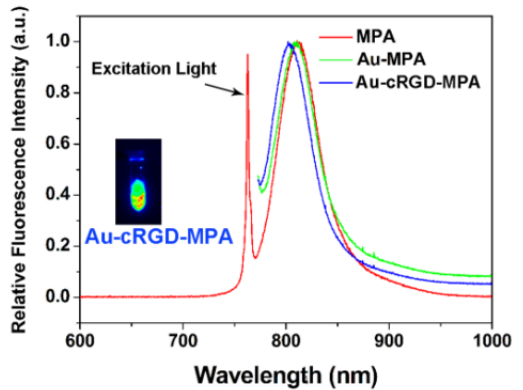

D

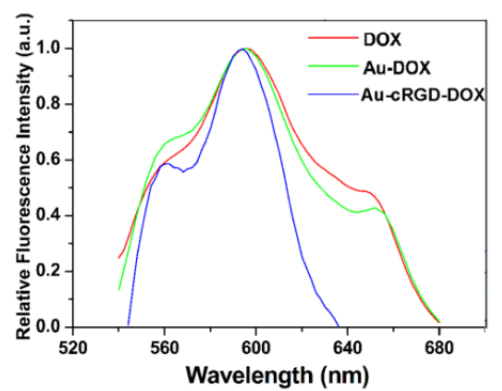

$\mathbf{F}$

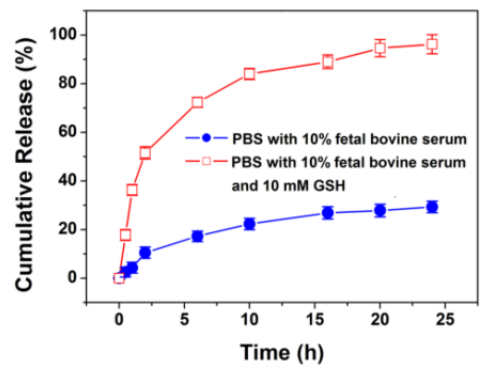

Fig 2. A) Absorption spectra of Au NS, MPA, Au-MPA and Au-cRGD-MPA; B) Fluorescence spectra of MPA, Au-MPA, Au-cRGD-MPA and florescence image of Au-cRGD-MPA collected by NIR fluorescence imaging system; C) Absorption spectra of Au NS, DOX, Au-DOX, Au-cRGD-DOX; D) Fluorescence spectra of DOX, Au-DOX, Au-cRGD-DOX; E) UV-Vis spectra of Au NS and Au-cRGD-DOX dispersed in fetal bovine serum immediately and after 8 h; F) The in vitro DOX release profile in PBS containing $10 \%$ fetal bovine serum with or without GSH (10 mM).

The results further demonstrate that the medium containing of the nanostructures (Au-MPA, Au-cRGD-MPA, Au-DOX and Au-cRGD-DOX) generated significant temperature increases upon excitation (Fig. 3A). For example, Au-cRGD-MPA and Au-cRGD-DOX attained a temperature of $38.7^{\circ} \mathrm{C}$ and $43.9^{\circ} \mathrm{C}$ in only $1 \mathrm{~min}$ and $5 \mathrm{~min}$, respectively. Similar$l y$, the result from the in vivo photo-thermal evaluation was consistent with the in vitro data (Fig. 3B). All the intratumoral temperature exceeded $43^{\circ} \mathrm{C}$ for $\mathrm{S} 180$ tumor-bearing mice treated with $\mathrm{Au} \mathrm{Ns}, \mathrm{Au}-\mathrm{DOX}$ and Au-cRGD-DOX and irradiated by NIR light for 10 min. On the contrary, no apparent temperature change was detected for PBS both in vitro and in vivo because of the low light absorption by the solution.

The GSH coated Au NS have both carboxyl and amine groups on the surface. Alhough slower centrifugation of $8000 \mathrm{rpm}$ for $15 \mathrm{~min}$ instead of 12000 rpm for $20 \mathrm{~min}$ was utilized to purify Au NS, the slight nanostars aggregation still persisted (Fig. 1C). This may be attributed to the cross linking induced by the coupling agents (EDC/NHS). The quenching effect of gold nanoparticles towards quantum dots and dye molecules had been widely reported [27-29]. However, our results demonstrated that the limited extent of overlap between absorption spectrum of $\mathrm{Au}$ NS and emission spectrum of MPA or DOX caused minimal quenching effect of Au NS. Simultaneously, the linkers with long chains could be anchored on the surfaces of $\mathrm{Au}$ NS, which may perturb the energy transfer by increasing the distance between Au NS and the attached molecule. In addition, quenching efficiency decreases with increasing nanoparticle diameter, which favors the relatively larger size of $\mathrm{Au}$ NS [30-32]. 


\section{Cell targeting capability of Au-cRGD-DOX}

To visualize the cellular uptake of DOX, Au-DOX and Au-cRGD-DOX, the red fluorescence emitted by DOX was captured by LCFM (Fig. 4A) after free DOX and the nanoconjugates were incubated with MDA-MB-231 and Bel-7402 tumor cells, which over-express integrin $\alpha_{v} \beta_{3}$, and MCF-7 tumor cells, which have low expression of integrin $\alpha_{v} \beta_{3}$. The weak red fluorescence of DOX in MCF-7 cells indicates that low amount of DOX, Au-DOX or Au-cRGD-DOX had entered tumor cells. Similarly, the amount of DOX or Au-DOX that entered the MDA-MB-231 cells and Bel-7402 cells was so low that there was no significant difference between the fluorescence intensity from these groups of cells and MCF-7 cells treated with DOX, Au-DOX, or $\mathrm{Au}-\mathrm{cRGD}-\mathrm{DOX}$. By contrast, Au-cRGD-DOX in MDA-MB-231 and Bel-7402 tumor cells showed ob-

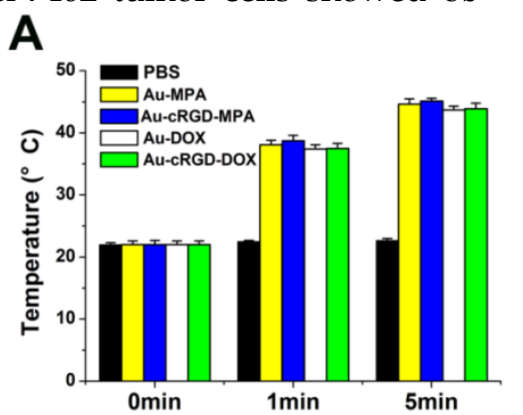

vious red fluorescence. The fluorescence intensity of the three cell lines incubated with different samples was compared (Fig. 4B). Significant differences appeared in MDA-MB-231 and Bel-7402 cells. Additionally, taking advantage of the "optical sectioning" function of LCFM, fluorescence images of MDA-MB-231 cells treated with Au-cRGD-DOX was reconstructed in three-dimensions (Fig. 4C). Obviously, Au-cRGD-DOX entered the cells, as evidenced by fluorescence signals appeared along the $\mathrm{Z}$ axis. All the results indicated clearly that the presence of cRGD on the surface of $\mathrm{Au}$ NS increased its cellular uptake by MDA-MB-231 and Bel-7402 cells. This result agrees with the work of Lee et al, [33] who demonstrated the targeted cellular uptake of cyclic RGD conjugated prodrugs via RGD-dependent endocytosis mechanisms.

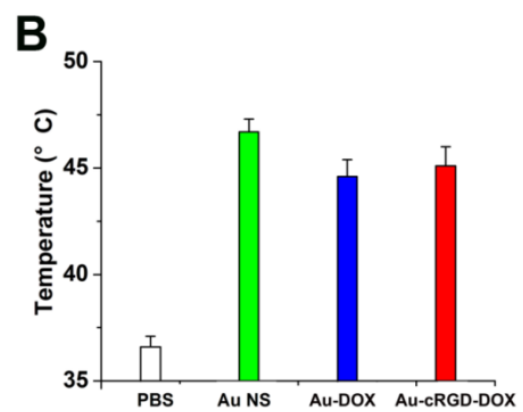

Fig 3. A) Temperature of PBS, Au-MPA, Au-cRGD-MPA, Au-DOX and Au-cRGD-DOX ( $50 \mu g / \mathrm{mL})$ in DMEM medium versus exposure time under I.0 W/cm 2 NIR light irradiation; B) The intratumoral temperature of SI 80 tumor-bearing mice after intratumoral injection of PBS, Au NS, Au-DOX and Au-cRGD-DOX upon light irradiation for $10 \mathrm{~min}$.

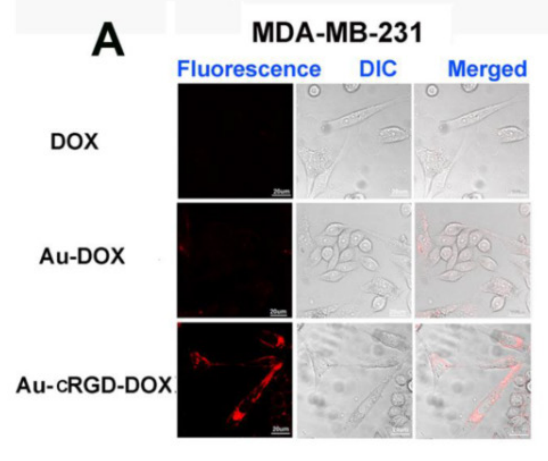

B

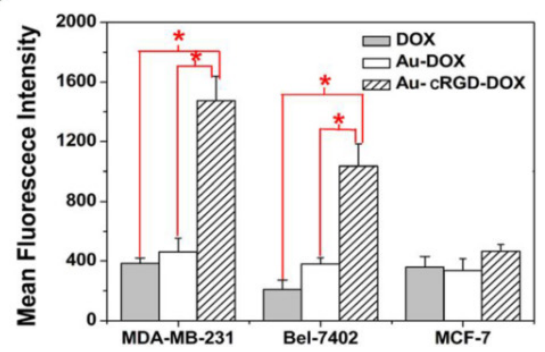

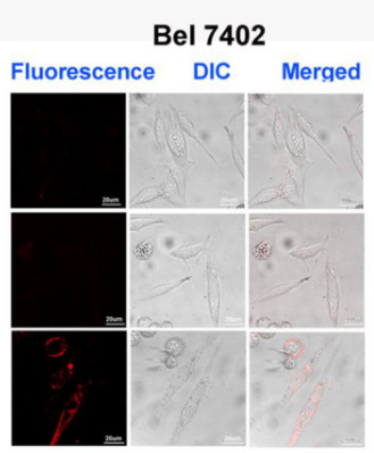

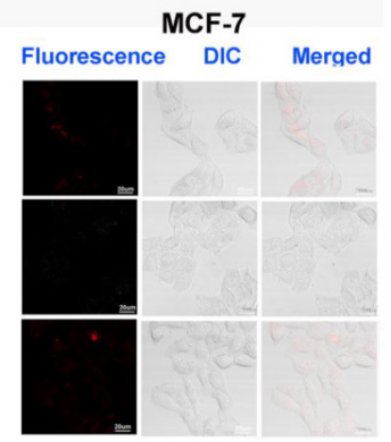

C

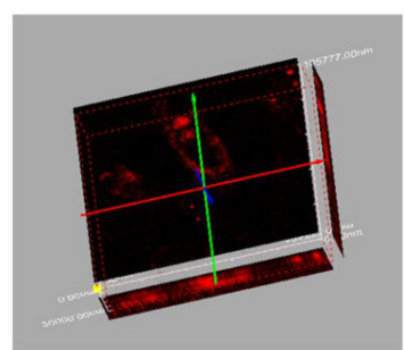

Fig 4. The tumor-targeting efficacy of DOX, Au-DOX and Au-cRGD-DOX in different cell lines. A) Intracellular uptake of DOX, Au-DOX, Au-cRGD-DOX MDA-MB-23I cells, Bel-7402 cells and MCF-7 cells respectively after incubation for I h; Scale bar $=20 \mu \mathrm{m}$ (objective: $20 \times$; zoom: $4 \times$ magnification). B) Mean red fluorescence intensity of different samples (DOX, Au-DOX, Au-cRGD-DOX) determined in MDA-MB-23I cells, Bel-7402 cells and MCF-7 cells; C) The reconstructed three-dimensional fluorescence image of MDA-MB-23I cells treated with Au-cRGD-DOX for I h. $(p<0.05)$. 
To further understand the intracellular kinetics of the multifunctional nanoparticles, the fluorescence images of Au-cRGD-DOX after incubating with MDA-MB-231 cells for a longer time $(8 \mathrm{~h})$ were also collected and Hoechst was used for nucleus staining (Fig. 5). The result clearly indicated that Au-cRGD-DOX or released DOX entered the nucleus, with only a small fraction remaining in the cytoplasm after $8 \mathrm{~h}$ incubation (Fig. 5B). The fluorescence images at different slices (Fig. 5A) and the three dimensional fluorescence image re-constructed from the slice images (Fig. 5C) further confirmed that Au-cRGD-DOX or released DOX reached the nucleus. Dam et al. [34] designed a nanostructure composed of nucleo- lin-specific aptamer and gold nanostars, which were actively transported to the nucleus after $5 \mathrm{~h}$ incubation, increasing significantly after $7 \mathrm{~h}$, and induced major changes to the nuclear phenotype. In another study by Yuan et al. [18] TAT-peptide functionalized gold nanostars were observed in the nuclear region by two-photon photoluminescence imaging. However, they did not find any true intranuclear TAT-NS, except some particles in the nuclear cleft. These results suggest that the red fluorescence observed from the nucleus in our study may be contributed by free DOX released from Au-cRGD-DOX as a result of the replacement of GSH on Au NS surface by the abundant intracellular GSH.

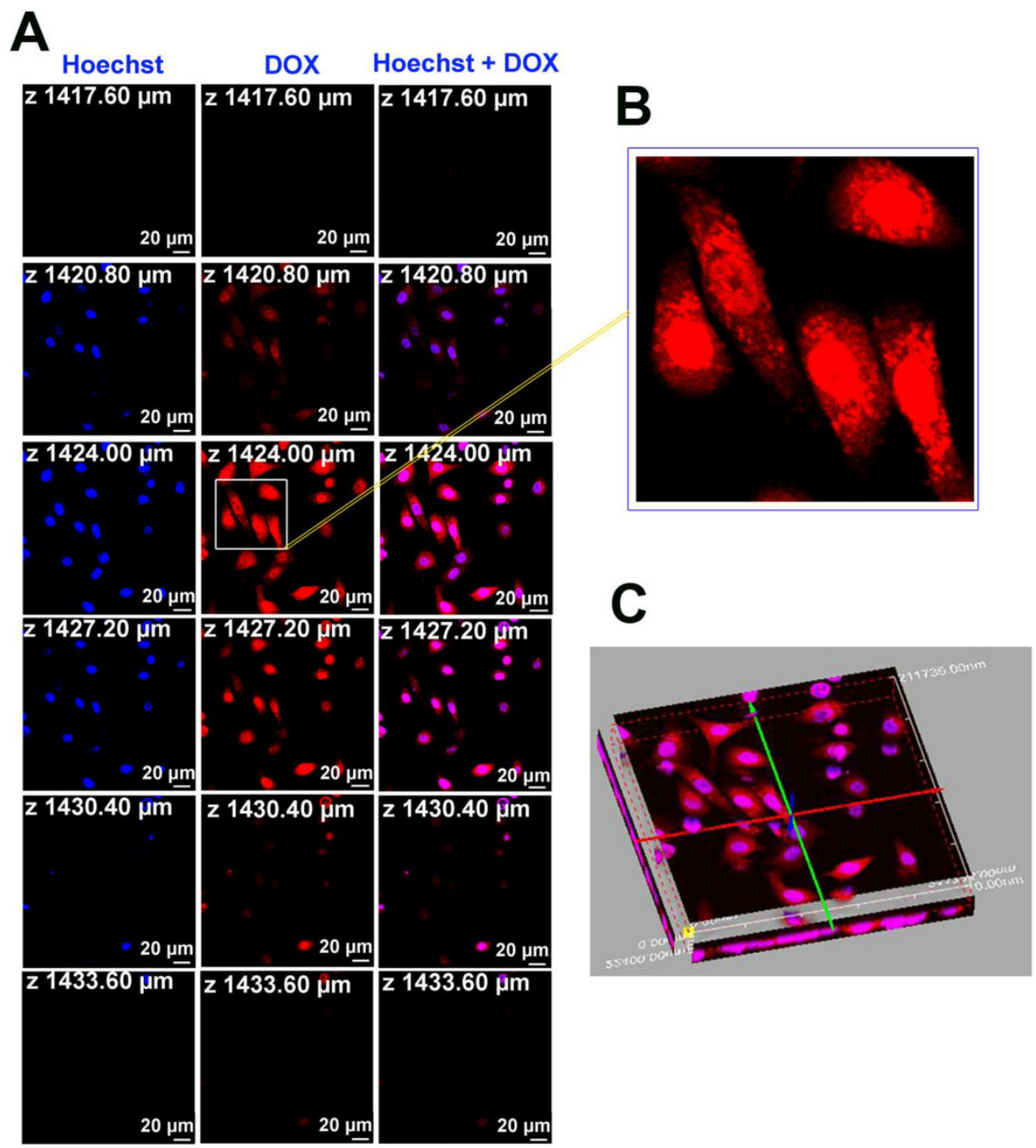

Fig 5. A) The fluorescence images of Au-cRGD-DOX after incubating with MDA-MB-23 I cells for $8 \mathrm{~h}$ at different optical slices (from I 4 I $7.60 \mu \mathrm{m}$ to $\mid 433.60 \mu \mathrm{m}$ ); B) Accumulation of Au-cRGD-DOX in the nucleus, indicated by the red fluorescence, was obviously observed from partial enlargement of the red fluorescence image at $Z=\mid 424.00 \mu \mathrm{m} ; \mathrm{C}) 3 \mathrm{D}$ fluorescence image of Au-cRGD-DOX reconstructed from Fig. 5A suggested that Au-cRGD-DOX were internalized by MDA-MB-23 I cells and are transported to the nuclei. 


\section{A}
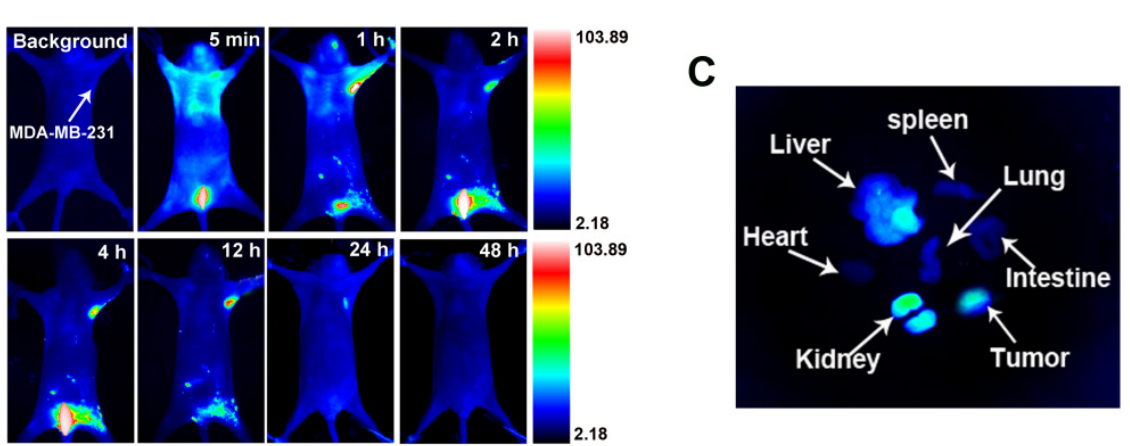

B
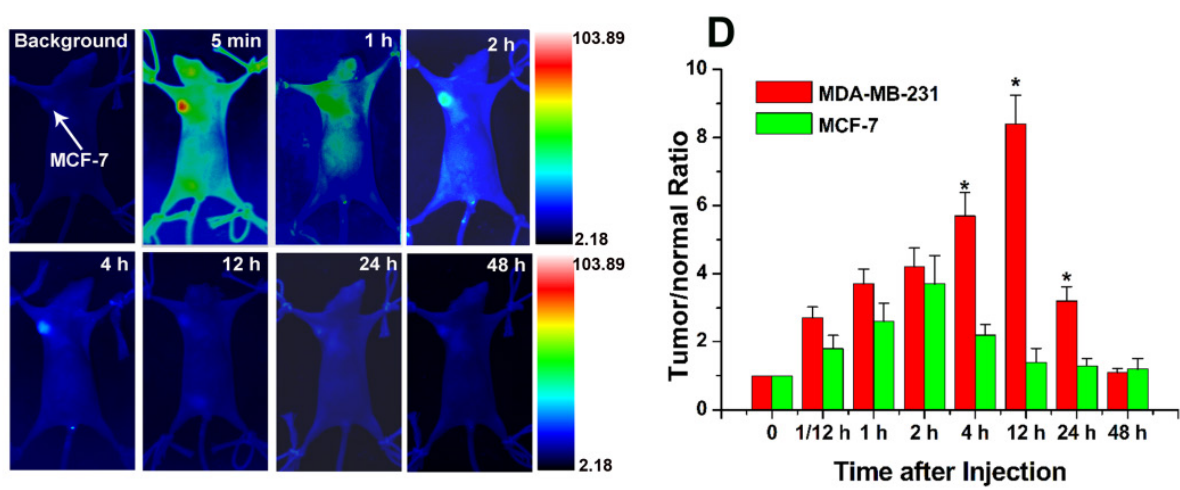

Fig 6. The biodistribution of Au-cRGD-MPA in different tumor-bearing mice models. In vivo fluorescence images of Au-cRGD-MPA in A) MDA-MB-23I (positive $\alpha_{v} \beta_{3}$ receptor expression) and B) MCF-7 (negative $\alpha_{v} \beta_{3}$ receptor expression) tumor-bearing mice model at different time intervals ( 5 min, I h, $2 \mathrm{~h}, 4 \mathrm{~h}, 12 \mathrm{~h}, 24 \mathrm{~h}$ and $48 \mathrm{~h}$ ); C) The ex vivo fluorescence images of isolated organs (heart, liver, spleen, lung, kidney, intestine and tumor) from the MDA-MB-23 I tumor-bearing mice at 48 $\mathrm{h}$ post-injection; the ex vivo fluorescence image of Au-cRGD-MPA in major organs (heart, liver, spleen, lung, kidney, intestine and tumor) detected at $48 \mathrm{~h}$ post-injection (MDA-MB-23I tumor-bearing mouse). D) Tumor-to-normal tissue (T/N) ratios of Au-cRGD-DOX in MDA-MB-23I and MCF-7 tumor-bearing mice at different post-injection time. $(p<0.05)$.

\section{Targeting ability of Au-cRGD-MPA in tumor bearing mice}

An important concern for nanoparticle-based treatments is whether the injected nanoparticles could preferentially accumulate in foci or can be cleared from the body. To trace the biodistribution and tumor targeting ability of the Au-cRGD, NIR fluorescence probe (MPA) was linked to the nanoconstruct for in vivo NIR imaging. Mice bearing MDA-MB-231 (integrin $\alpha_{v} \beta_{3}$ positive) tumors and MCF-7 tumors (low integrin $\alpha_{v} \beta_{3}$ expression) were selected for the animal models. Series of in vivo NIR fluorescence images at different time intervals show that Au-cRGD-MPA initially distributed all over the body, and subsequently cleared by the hepatobiliary and renal pathways in MDA-MB-231 tumor-bearing mice (Fig. 6A). The fluorescence signal in MDA-MB-231 tumor was distinguishable from the normal tissues at $1 \mathrm{~h}$ post-injection, reaching peak intensity at $12 \mathrm{~h}$ post-injection before gradually disappearing within 48. In contrast, MCF-7 tumor uptake in mice treated with Au-cRGD-MPA became visible after $2 \mathrm{~h}$ post-injection and rapidly disappeared from $4 \mathrm{~h}$ post-injection, which represented the contribution of enhanced permeation and retention in the tumor by the nanoconstructs (Fig. 6B). The ex vivo fluorescence images of the excised organs separated from MDA-MB-231 tumor-bearing mice confirmed that the nanoconstructs prominently accumulated in tumor and reticuloendothelial system of the liver even after the long circulation (Fig. 6C). To semi-quantitatively analyze the targeting efficacy of the probes for different tumors, fluorescence intensity of the tumors and normal tissues was analyzed at different time intervals (Fig. 6D). The fluorescence intensity ratio between MDA-MB-231 tumor and normal tissue $(\mathrm{T} / \mathrm{N})$ was 3.7 at $1 \mathrm{~h}$ post-injection of the probe, reaching a peak of 8.4 at $12 \mathrm{~h}$ post-injection, and decreasing to 1.1 at $48 \mathrm{~h}$. For MCF-7 tumor-bearing mice, $\mathrm{T} / \mathrm{N}$ was 2.6 at $1 \mathrm{~h}$ post-injection, reaching a peak of 3.7 at $2 \mathrm{~h}$ post-injection, and rapidly declining to 1.4 at $12 \mathrm{~h}$.

Although several research groups have investigated the intracellular behavior of the gold nanostars by different techniques including multi-photon microscopy [35], confocal fluorescence microscopy [34], and high resolution TEM [18], the biodistribution of the gold nanostars in living subjects was still unknown. In this work, NIR fluorescence imaging, pos- 
sessing the advantages of effective, low-cost, non-invasive and flexible features, was utilized to in vivo evaluate the tumor-targeting capability of Au-RGD. A derivative of FDA approved NIR fluorescence probe (MPA), which was provided with carboxyl group, was selected to conjugate with $\mathrm{Au}$ NS. The in vivo fluorescence imaging results indicated the high affinity and specificity of cRGD to integrin $\mathrm{a}_{\mathrm{v}} \beta_{3}$ in MDA-MB-231 mouse model, which was consistent with the tumor-targeting evaluation of $\mathrm{Au}-\mathrm{RGD}$ in cells. Given the in vivo fluorescence imaging results, Au-RGD could serve as the drug carrier or therapy agents by selectively accumulating in high $\mathrm{a}_{\mathrm{v}} \beta_{3}$ expression tumors in biological system. In addition, the renal clearance observed by in vivo (Fig. 6A) and in vitro (Fig. 6C) imaging suggests that some of the dyes (MPA) were detached from Au NS surface in the blood circulation. Replacement of GSH in vitro or in vivo by other thiol-containing biomolecules will affect the imaging and therapy results. This potentially deleterious effect could be avoided in future by using more stable thiol-containing molecules to bind with Au NS.

\section{Anti-tumor efficiency of Au-cRGD-DOX}

Therapeutic effects of Au-cRGD-DOX at cell level

To confirm the enhancement of anti-tumor efficacy of Au-cRGD-DOX, cytotoxicity effects of $\mathrm{Au}$ NS, free DOX, the photothermal effect of Au NS under $765 \mathrm{~nm}$ laser irradiation for $10 \mathrm{~min}$, and the combined chemo- and photothermal effects of Au-DOX and Au-cRGD-DOX under $765 \mathrm{~nm}$ laser irradiation for $10 \mathrm{~min}$ were compared by evaluating the number and morphological changes of MDA-MB-231, Bel-7402, and MCF-7 tumor cells at $4 \mathrm{~h}$ after incubation with the five different samples. As shown in Fig. 7A, different groups of cells showed diverse degrees of apoptosis. For the groups incubated with Au NS alone, the majority of the cells maintained their original morphology. Compared to the group of cells incubated with DOX or Au NS with laser irradiation, more obvious cellular morphological changing (became rounded) characteristic of apoptosis could be observed among MCF-7, MDA-MB-231 and Bel-7402 tumor cells, which have been incubated with Au-DOX or Au-cRGD-DOX and exposed to laser irradiation. Furthermore, there was no obvious difference between the two groups of MCF-7 cells treated with Au-DOX and Au-cRGD-DOX. In comparison with the groups treated with Au-DOX, it is evident that much less MDA-MB-231 cells and Bel-7402 cells were left in dishes after the chemophotothermal treatment of Au-cRGD-DOX, which indicated en- hanced anti-tumor efficacy of Au-cRGD-DOX towards tumor cells with high expression of integrin $\mathrm{a}_{\mathrm{v}} \beta_{3}$. Additionally, in vitro anti-tumor efficacy of $\mathrm{Au}$-cRGD-DOX was also demonstrated by quantitatively evaluating its cytotoxicity effect via MTT assay on MDA-MB-231 tumor cells (Fig. 7B). All the samples showed dose-dependent anti-tumor activity. At the highest dose level, the inhibition ratio of Au-cRGD-DOX was over $90 \%$ of total cells, which was much higher than that of Au NS (68\%), DOX $(55 \%)$ and Au-DOX (80 \%) at the same dose level.

With the increase of the concentration of Au-cRGD-DOX, the cell viability distinctively decreased after incubation for $4 \mathrm{~h}$, which correlated with Annexin V-FITC and PI staining (Fig. 8A and Fig. 8B). It was easy to distinguish non-apoptotic cells (no fluorescence), early apoptotic cells (green fluorescence), late apoptotic cells (green and red fluorescence) and necrotic cells (red fluorescence) [36]. When MDA-MB-231 cells were incubated with $\mathrm{Au}-\mathrm{cRGD}-\mathrm{DOX}$, red fluorescence in nucleus appeared after 10 min exposure, which suggests the destruction of the cell membrane integrity and enzymes used in DNA synthesis. Nevertheless, only green fluorescence and less red dots were detected for other groups, which demonstrated that MCF-7 cells and MDA-MB-231 cells treated with Au NS, DOX and $\mathrm{Au}$-DOX were in the early apoptotic period. The green fluorescence intensity and red fluorescence intensity at different groups were also compared (Fig. 8C and Fig. 8D). Obviously, Au-cRGD-DOX incubated MDA-MB-231 cells possessed the strongest red fluorescence after NIR light excitation. However, green fluorescence intensity in other groups far exceeded the red fluorescence intensity. These results illustrate that the tumor cells with positive expression of integrin $\alpha_{v} \beta_{3}$ have distinctly superior therapeutic efficacy than those cells with low expression of RGD receptors.

Most normal cells as well as some integrin $\alpha_{v} \beta_{3}$ expression-negative tumor cells such as MCF-7 breast tumor cells, could take up small amounts of Au-cRGD-DOX, resulting in low chemophotothermal effect from Au-cRGD-DOX [37]. Thus, the cyclic RGD peptide on the surface of Au NS recognizes integrin $\alpha_{v} \beta_{3}$ expression-positive tumor cells, and mediates internalization of the nanoconjugates in cells. Subsequent endosomal transport and lysosomal degradation could release the toxic DOX from Au-cRGD-DOX to induce apoptosis in cancer cell. Similarly, $\mathrm{Xu}$ et al. [38] have demonstrated the improved photothermal effect of combining RGD-conjugated gold nanorods that target integrin $\alpha_{v} \beta_{3}$ with irradiation of melanoma cancer cells. Xiao et al. [39] have used the gold nano- 
rods conjugated with DOX and cRGD for combined anticancer delivery. However, to the best of our knowledge, we are not aware of research works combining the photothermal therapeutic effect of gold nanostars and the chemotherapeutic effect of DOX for tumor-targeted therapy. The results herein demonstrate the selective anti-tumor efficacy of Au-cRGD-DOX combined chemotherapy and the photothermal therapy under NIR laser irradiation could eclipse that of DOX, Au NS, or Au-DOX.

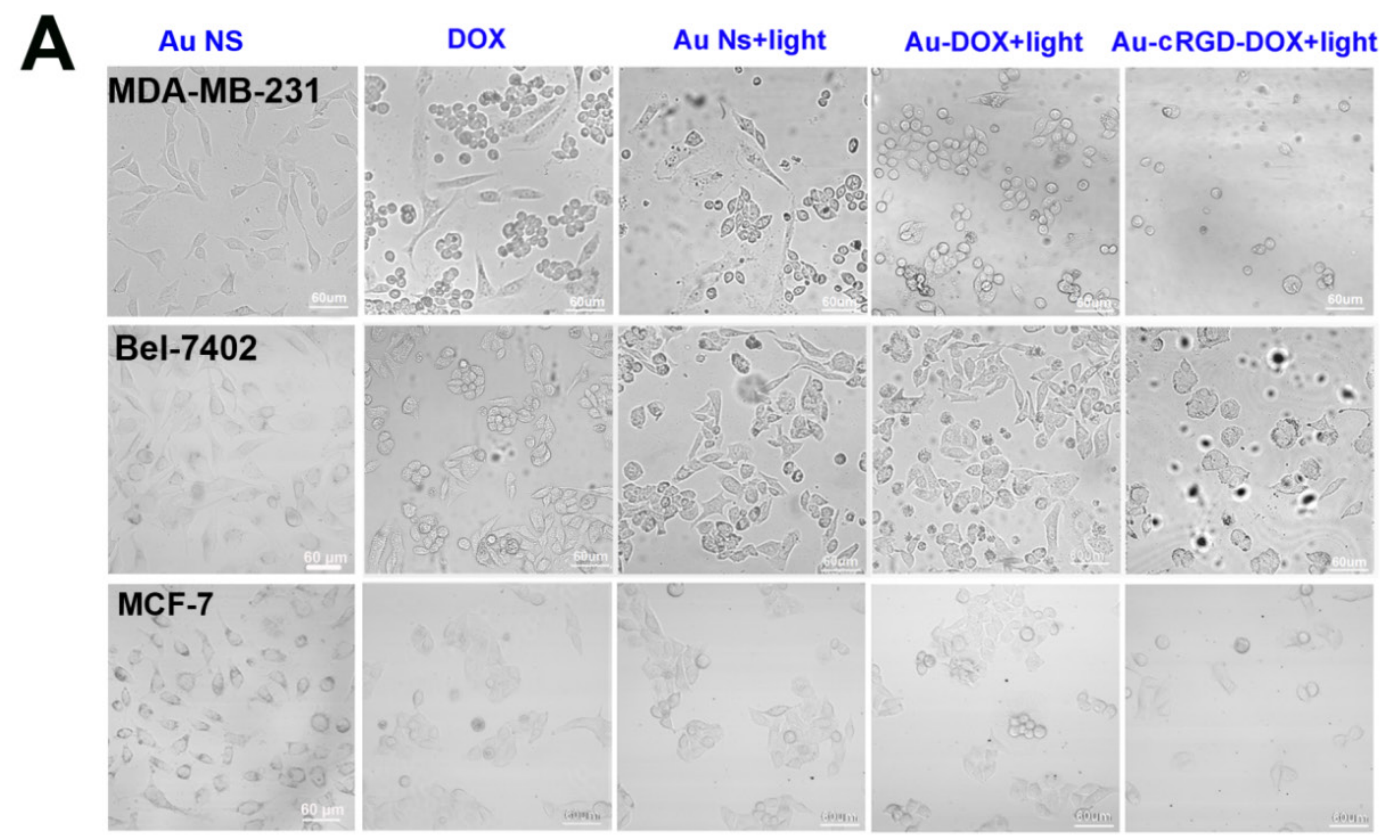

B

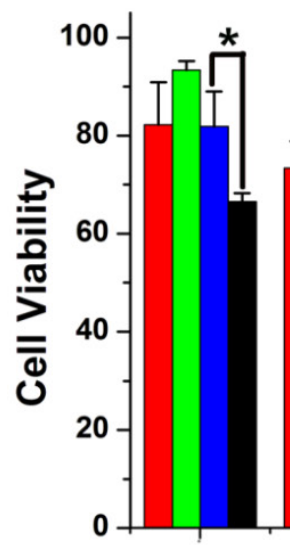

Au Ns: 0.7

Conjugated DOX: 0.02

Free DOX: 0.02
0.1

0.1

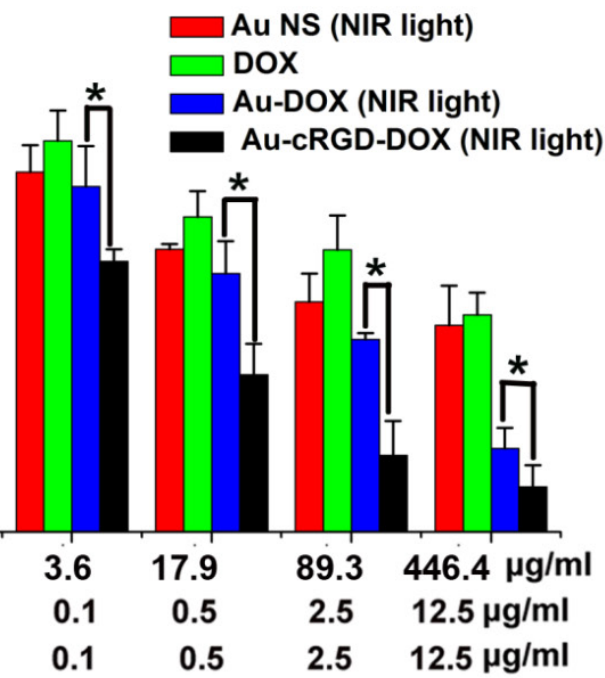

Fig 7. A) The morphological changes of MDA-MB-23I, Bel-7402 and MCF-7 tumor cells at $4 \mathrm{~h}$ after the treatment of four different samples (Au NS, DOX, Au NS + light, Au-DOX + light, Au-cRGD-DOX + light) were compared; B) MTT assay was utilized to qualitatively display in vitro anti-tumor activity of Au NS, free DOX, Au-DOX and Au-cRGD-DOX on MDA-MB-23I tumor cell. Au-cRGD-DOX treated cells demonstrated the lowest cell viability (39.67 \%) upon irradiated by the NIR light. $(p<0.05)$ 

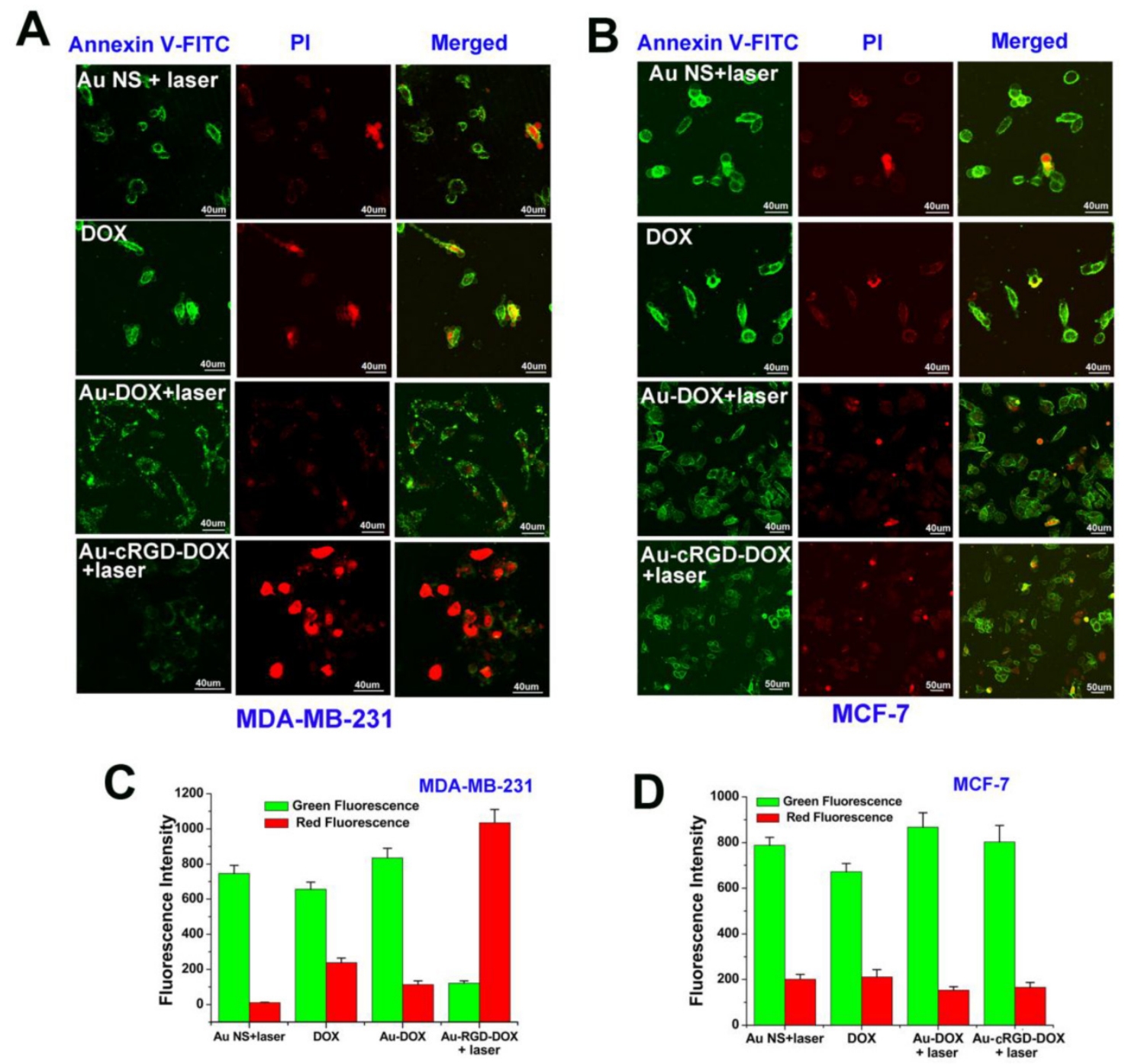

Fig 8. Annexin V-FITC and PI staining were used to evaluate the cell viability following treatment of different samples. A) Morphologic changes of MDA-MB-23I and MCF-7 cell apoptosis after administration of the four sample (Au NS + light, DOX, Au-DOX + light, Au-cRGD-DOX + light) were compared by cell apoptosis probes staining and detected by confocal laser scanning microscope. Au-cRGD-DOX incubated MDA-MB-23I cells possessed the strongest red fluorescence after NIR light excitation. The mean red and green fluorescence intensity of B) MDA-MB-23I cells and C) MCF-7 cells after treatment of different samples were collected after staining of the double cell apoptosis probes. $(P<0.05)$.

\section{Therapy evaluation of Au-cRGD-DOX in tu- mor-bearing mice}

Although multifunctional gold nanoparticles have been studied for targeted chemo- and photothermal cancer treatment at cell level [40,41], limited work has been done to compare their therapeutic effect in animals. Herein, the anti-tumor efficacy of Au-cRGD-DOX was investigated in S180 tumor-bearing mice. We compared the therapeutic effect from different administration routes (intratumoral or tail vein injection). Considering the limited bioavailability of the drugs via tail vein injection, the intravenous dose was selected as 1.5 times as the intratumoral injection dose. The images of tumor-bearing mice at 9 and 18 days post-injection are shown in Figure S4 (Supplementary Material), after injecting the animals with saline, DOX, Au NS, Au-DOX, and Au-cRGD-DOX via intratumoral or tail vein injection combined with laser irradiation. Tumors of the mice that received the photothermal and chemo-therapy from Au-cRGD-DOX via intratumoral or tail vein injection were similar to each other. However, they were obviously much smaller than those of other groups. In order to quantitatively analyze the therapeutic effect of samples, the survival rate, tumor volume, and body weight of each group of mice were recorded at the interval of 3 days intervals (Fig. 9A, Fig. 9B and Fig. 9C). The mice treated with Au-cRGD-DOX via intravenous injection and irradiation with NIR laser maintain a $100 \%$ survival rate after 28 days, which is higher than the mice treated with 
Au-cRGD-DOX via tail vein (87.5 \%). The survival rate of DOX and saline treated mice were $75 \%$ and 50 $\%$, respectively (Fig. 9A). As shown in Fig. 9B, tumor volume belonging to the mice treated with $\mathrm{DOX}, \mathrm{Au}$ NS and Au-DOX are three to five folds larger than the mice treated with Au-cRGD-DOX. Furthermore, the tumor volume of Au-cRGD-DOX treated mice via tail vein injection is similar to that of Au-cRGD-DOX treated mice via intratumoral injection. The body weight of each group increased proportionately during the observation period (Fig. 9C). The mice treated with saline had the lowest body weight in comparison with the mice in other groups. To further compare the anti-cancer efficiency by tail vein and intratumoral injection, ex vivo histology studies were performed (Fig. 9D). Significant necrosis occurred in the peripheral and central tumor region for intratumoral injection group, and only a small amount of tumor cells appeared around the tumor vessels. For tail vein injection group, there were many cancer nests with clear boundary and many highly proliferating tumor cells. Moreover, much smaller necrotic region with pathological calcification was observed in the tumor-bearing mice injected by tail vein.
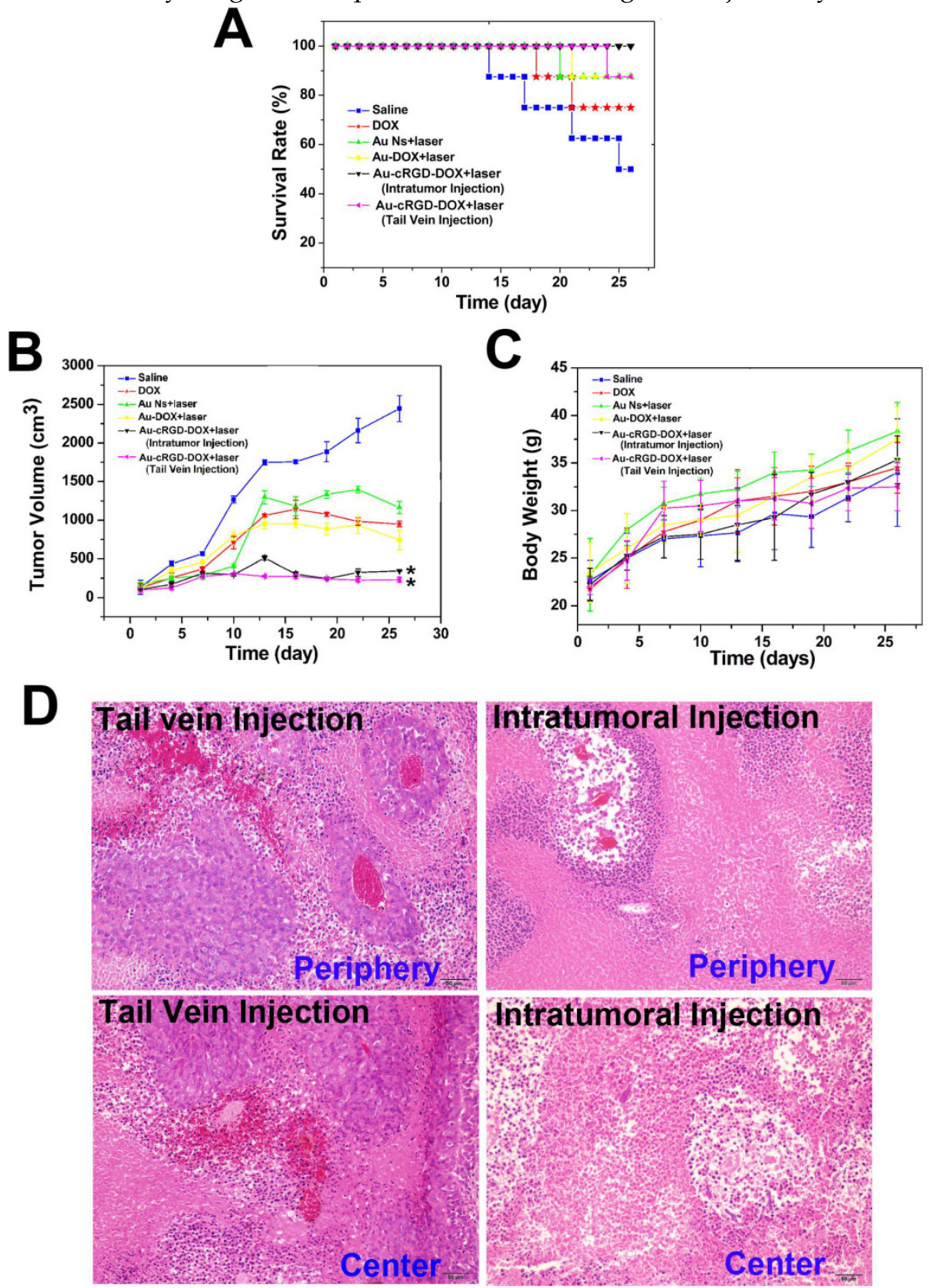

Fig 9. Comparison of the therapeutic efficacy of Au NS + light, Au-DOX + light, free DOX, Au-cRGD-DOX+ light (intratumoral injection) and Au-cRGD-DOX + light (tail vein injection) in SI 80 tumor-bearing mice. A) Survival rates of mice in different treatment groups within 26 days; B) Tumor growth of the mice in different groups during treatment; C) Body weight changes of the mice in different treatment groups within 26 days; D) H\&E stained tumor tissue section (periphery and center) of Au-cRGD-DOX treated mice at 16 days after tail vein injection or intratumoral injection. $(P<0.05)$. 
$\mathrm{Au}$ NS has plasmon bands tunable in the NIR tissue optical window. These nanoparticles are very stable, with high potential to effectively transduce absorbed laser energy into heat [42]. The use of CW laser irradiation prevents the photo-thermal melting of $\mathrm{Au} \mathrm{Ns}$ nanostructures into gold nanospheres/aggregates within seconds after exposure of the nanostructures to femtosecond pulse lasers [43]. More importantly, the surface roughness and very high surface-to-volume ratio of $\mathrm{Au}$ NS favor high drug loading efficiency for anti-cancer drug delivery. Therefore, multi-functional anti-cancer nanoconstructs were constructed by covalently conjugating $\mathrm{Au}$ NS with targeting moiety (cRGD) and anti-cancer drug (DOX). A synergistic effect between the chemotherapy and thermotherapy was confirmed by the therapeutic results of Au-cRGD-DOX treated groups when compared with Au NS or free DOX treated groups. Meanwhile, the nature of targeting ligands can dramatically change the nanoparticle distribution in living subjects and the active targeting ligands did improve the total tumor uptake of nanoparticle, which was demonstrated by the different therapeutic results with Au-cRGD-DOX and Au-DOX. Huang et al. [44] suggested that the active molecular targeting of the tumor microenvironments does not significantly improve the total tumor accumulation of nanoparticles for long circulation and rigid gold nanoparticles. The work further suggested that intratumoral instead of intravenous injection is the preferred route of gold nanorods administration. Accordingly, we selected a 1.5 times dose for intravenous injection to compensate the loss of the drug in the body circulation, and tried to find out which of the two administration routes is a favorable one to be selected in tumor-targeting therapy. Our results showed that both routes of administration had similar outcomes including survival rate, tumor volume and body weight. Clearly, this reflects the rapid response of the S180 tumor model used to therapy. However, differences begins to emerge between the two routes of administration Day 25, where the intratumoral group retained $100 \%$ survival rate but significant decrease began to emerge for the intravenous group. Consequently, the similarity in the survival, tumor volume, and weight gain between the two routes of administration is a short-term effect that is expected to diverge at longer time points. The size of the particles is expected to influence the region of photothermal effect in the tumor. Intratumoral injection will deliver higher concentration of the multifunctional nanoparticle to the tumor than intravenous injection, which relies on both enhanced retention and permeation as well as receptor mediated uptake by the tumor. Effec- tive local concentration, therefore, depends on the amount of materials that reaches the target tissue, which is expected to be smaller for intravenous than intratumoral injection. Although the global response appeared to be similar for both routes of administrations, we postulated that the local therapeutic effects will be different.

Subsequently, we used histologic analysis to compare the anti-cancer efficiency by tail vein and intratumoral injection ex vivo. Tumors from intratumoral injection group exhibited much larger necrotic region than those from tail vein injection both in the peripheral or central region. This is expected because of the higher concentration of the nanoparticles in tumors by direct tumor injection. Extended survival studies will demonstrate the long term benefits of the intratumoral over intravenous administration route. Although our result suggests that intratumoral injection is a potentially effective therapeutic method, it is not a clinically preferred route. Therefore, efforts will be made to optimize the intravenous method of administration in future.

\section{Conclusion}

In summary, we have successfully developed tumor targeted Au-cRGD-MPA and Au-cRGD-DOX nanoconstructs for tumor imaging and combined photothermal and chemo-therapy in vivo. By taking advantage of NIR probe, we have directly visualized the biodistribution of Au-cRGD in tumor-bearing mice model. Tumor-targeting ability of the nanoconstructs was dramatically enhanced in integrin $\mathrm{a}_{\mathrm{v}} \beta_{3}$-overexpressing tumors by $\mathrm{a}_{\mathrm{v}} \beta_{3}$ mediated active targeting. Au-cRGD-DOX was actively delivered in close proximity to the nucleus, and then entered the nucleus after longer incubation time. The tumor cells showed significant morphological changes and apoptosis upon NIR light irradiation. In comparison with conventional hyperthermia or chemotherapy, the enhanced tumor therapy efficacy was demonstrated in Au-cRGD-DOX treated mice due to the combination of the dual treatment ways. Together, our results verified that the curative effect of the multifunctional nanoconjugates delivered by intratumoral injection had a decided advantage over the tail vein injection. The above factors provide insight into the development of new strategies to design drug-loaded nanoconstructs with increased therapeutic efficacy and prompt further exploration of these nanoconstructs for combined therapy of other related diseases.

\section{Abbreviations}

Au NS: gold nanostars; cRGD: cyclic RGD; NIR: near infrared; DOX: Doxorubicin; SPR: surface plas- 
mon resonance; PPTT: plasmonic photothermal therapy; RGD: arginine-glycine-aspartic acid; ICG: indocyanine green; HEPES: 4-(2-hydroxyethyl)-1piperazineethane-sulfonic acid; GSH: reduced glutathione; EDC: 1-ethyl-3-[3-diMethylaminopropyl] carbodiimide hydrochloride; DCC: N, N'-Dicyclohexylcarbodiimide; NHS: N-hydroxysuccinimide; MTT: 3-(4, 5- diMethylthialzol-2-yl)-2, 5-diphe-nyltetrazolium bromide; FBS: fetal bovine serum; Au-GSH: Au-Glutathione; Au-cRGD-DOX: Au-cRGD-Doxorubicin; TEM: transmission electron microscope; LCFM: laser confocal fluorescence microscope; ROI: region of interest; DIC: differential interference contrast; $\mathrm{T} / \mathrm{N}$ : tumor to normal tissue contrast ratios.

\section{Supplementary Material}

Fig.S1 - S5. http:// www.thno.org/v03p0633s1.pdf

\section{Acknowledgments}

The authors are grateful to Natural Science Foundation Committee of China (NSFC 81000666, 81220108012, 81211140243 and 81171395, the Program for New Century Excellent Talents (NCET) in University of the Ministry of Education of China, and the Priority Academic Program Development of Jiangsu Higher Education Institutions for their financial support.

\section{Competing Interests}

The authors have declared that no competing interest exists.

\section{References}

1. Jaffray DA. Image-guided radiotherapy: from current concept to future perspectives. Nat Rev Clin Oncol. 2012; 9: 688-99.

2. Li SW, Wang H, Liu ML, Zhang HB, Xiang YQ, Lv X, et al. Positive effect of high RKIP expression on reduced distant metastasis by chemotherapy when combined with radiotherapy in locoregionally advanced nasopharyngeal carcinoma: a prospective study. Med Oncol. 2013; 30: 322-7.

3. Mi Y, Guo Y, Feng SS. Nanomedicine for multimodality treatment of cancer. Nanomedicine. 2012; 7: 179-4.

4. Mi Y, Liu X, Zhao J, Ding J, Feng SS. Ultimodality treatment of cancer with herceptin conjugated, thermomagnetic iron oxides and docetaxel loaded nanoparticles of biodegradable polymers. Biomaterials. 2012; 33: 7519-29.

5. Kennedy LC, Bickford LR, Lewinski NA, Coughlin AJ, Hu Y, Day ES, et al. A new era for cancer treatment: gold-nanoparticle-mediated thermal therapies. Small. 2011; 7: 169-83.

6. Weissleder R. A clearer vision for in vivo imaging. Nat Biotechnol. 2001; 19: 316-7.

7. Yi DK, Sun IC, Ryu JH, Koo H, Park CW, Youn IC, et al. Matrix metalloproteinase sensitive gold nanorod for simultaneous bioimaging and photothermal therapy of cancer. Bioconjug Chem. 2010; 21: 2173-7.

8. Choi WI, Kim JY, Kang C, Byeon CC, Kim YH, Tae G. Tumor regression in vivo by photothermal therapy based on gold-nanorod-loaded, functional nanocarriers. ACS Nano. 2011; 5: 1995-2003.

9. Diagaradjane P, Shetty A, Wang JC, Elliott AM, Schwartz J, Shentu S, et al. Modulation of in Vivo Tumor Radiation Response via Gold Nanoshell-Mediated Vascular-Focused Hyperthermia: Characterizing an Integrated Antihypoxic and Localized Vascular Disrupting Targeting Strategy. Nano Lett. 2008; 8: 1492-1500.
10. Chen JY, Charles G, Richard L, Zhang Q, Yang MX, Michael G, et al. Gold Nanocages as Photothermal Transducers for Cancer Treatment. Small. 2010; 6: 811-7.

11. Lu W, Xiong C, Zhang G, Huang Q, Zhang R, Zhang JZ, et al. Targeted photothermal ablation of murine melanomas with melanocyte-stimulating hormone analog-conjugated hollow gold nanospheres. Clin Cancer Res. 2009; 15: 876-86.

12. Van de Broek B, Devoogdt N, D'Hollander A, Gijs HL, Jans K, Lagae L, et al. Specific cell targeting with nanobody conjugated branched gold nanoparticles for photothermal therapy. ACS Nano. 2011; 5: 4319-28.

13. Huang XH, Jain PK, EI-Sayed IH, EI-Sayed MA. Plasmonic phtotothermal therapy (PPTT) using gold nanoparticles. Laser Med Sci. 2008; 23: 217-28

14. Feng H, Colleen LN, Jason HH, Peter N. Plasmon Resonances of a Gold Nanostar. Nano Lett. 2007; 7: 729-32.

15. Sironi L, Freddi S, Caccia M, Pozzi P, Rossetti L, Pallavicini P. Gold Branched Nanoparticles for Cellular Treatments. J Phys Chem C. 2012; 116: 18407-18.

16. Smith AM, Mancini MC, Nie S. Bioimaging: Second window for in vivo imaging. Nat Nanotechnol. 2009; 4: 710-1.

17. Halas NJ. Plasmonics: An Emerging Field Fostered by Nano Letters. Nano Lett. 2010; 10: 3816-22.

18. Yuan H, Fales AM, Vo-Dinh T. TAT peptide-functionalized gold nanostars: enhanced intracellular delivery and efficient NIR photothermal therapy using ultralow irradiance. J Am Chem Soc. 2012; 134: 11358-61.

19. Kim YH, Jeon J, Hong $\mathrm{SH}$, Rhim WK, Lee $\mathrm{YS}$, Youn $\mathrm{H}$, et al. Tumor Targeting and Imaging Using Cyclic RGD-PEGylated Gold Nanoparticle Probes with Directly Conjugated Iodine-125. Small. 2011; 7: 2052-60.

20. Seftor RE. Role of the $\beta 3$ integrin subunit in human primary melanoma progression: multifunctional activities associated with $\alpha(v) \beta 3$ integrin expression. Am J Pathol. 1998; 153: 1347-51.

21. Choi HS, Gibbs SL, Lee JH, Kim SH, Ashitate Y, Liu F, et al. Targeted zwitterionic near-infrared fluorophores for improved optical imaging. Nat Biotechnol. 2013; 6: 148-53.

22. Zhou Y, Kim YS, Lu X, Liu S. Evaluation of 99mTc-labeled cyclic RGD dimers: impact of cyclic RGD peptides and 99mTc chelates on biological properties. Bioconjug Chem. 2012; 23: 586-95.

23. Xie JP, Lee JY, Wang DIC. Seedless, Surfactantless, High-Yield Synthesis of Branched Gold Nanocrystals in HEPES Buffer Solution. Chem Mater 2007; 19: 2823-30.

24. Choi J, Yang J, Bang D, Park J, Suh JS, Huh YM, et al. Targetable gold nanorods for epithelial cancer therapy guided by near-IR absorption imaging. Small. 2012; 8: 746-53.

25. Good NE, Izawa K. Hydrogen ion buffers. Methods Enzymol. 1972; 2: 53-68.

26. Dam DH, Lee JH, Sisco PN, Co DT, Zhang M, Wasielewski MR, et al. Direct observation of nanoparticle-cancer cell nucleus interactions. ACS nano. 2012; 6: 3318-26.

27. Jang B, Park JY, Tung CH, Kim IH, Choi Y. Gold Nanorod-Photosensitizer Complex for Near-Infrared Fluorescence Imaging and Photodynamic/Photothermal Therapy In Vivo. ACS Nano. 2011; 5: 1086-94.

28. Jin LH, Li SM, Kwon BJ, Cho YH. Quenching dynamics in CdSe/ZnS core/shell quantum dots-gold nanoparticle conjugates in aqueous solution. Appl Phys. 2011; 109: 124310-5.

29. Yang PJ, Chu HC, Lee YH, Kobayashi T, Chen TC, Lin HC. Quenching effects of gold nanoparticles in nanocomposites formed in water-soluble conjugated polymer nanoreactors. Polymer. 2012; 53: 939-46.

30. Dulkeith E, Ringler M, Klar TA, Feldmann J, Muñoz Javier A, Parak WJ. Gold Nanoparticles Quench Fluorescence by Phase Induced Radiative Rate Suppression. Nano Lett. 2005; 5: 585-9.

31. Kim CK, Kalluru RR, Singh JP, Fortner A, Griffin J, Darbha GK, et al. Gold-Nanoparticle-Based Miniaturized Laser-Induced Fluorescence Probe for Specific DNA Hybridization Detection: Studies on Size-Dependent Optical Properties. Nanotechnology. 2006; 17: 3085-93.

32. Cobley $\mathrm{CM}, \mathrm{Au} \mathrm{L}, \mathrm{Chen} \mathrm{J}, \mathrm{Xia}$ Y. Targeting gold nanocages to cancer cells for photothermal destruction and drug delivery. Expert Opin Drug Deliv 2010;7:577-87.

33. Lee MH, Kim JY, Han JH, Bhuniya S, Sessler JL, Kang C, et al. Direct fluorescence monitoring of the delivery and cellular uptake of a cancer-targeted RGD peptide-appended naphthalimide theragnostic prodrug. J Am Chem Soc. 2012; 134: 12668-74.

34. Dam DH, Lee JH, Sisco PN, Co DT, Zhang M, Wasielewski MR, et al. Direct Observation of Nanoparticle-Cancer Cell Nucleus Interactions. ACS Nano. 2012; 6: 3318-26. 
35. Yuan H, Khoury CG, Wilson CM, Grant GA, Bennett AJ, Vo-Dinh T. In vivo particle tracking and photothermal ablation using plasmon-resonant gold nanostars. Nanomedicine. 2012; 8: 1355-63.

36. Span LF, Pennings AH, Vierwinden G, Boezeman JB, Raymakers RA, de Witte $\mathrm{T}$. The dynamic process of apoptosis analyzed by flow cytometry using Annexin-V/propidium iodide and a modified in situ end labeling technique. Cytometry. 2002; 47: 24-31.

37. Yun GE, Bin K, Ge Y, Kang B. Surface plasmon resonance scattering and absorption of biofunctionalized gold nanoparticles for targeted cancer imaging and laser therapy. Sci China Tech Sci. 2011; 54: 2358-62.

38. Xu WC, Luo T, Li P, Zhou CQ, Cui DX, Pang B, et al. RGD-conjugated gold nanorods induce radiosensitization in melanoma cancer cells by downregulating av $\beta 3$ expression. Int J Nanomedicine. 2012; 7: 915-24.

39. Xiao Y, Hong H, Matson VZ, Javadi A, Xu W, Yang Y, et al. Gold Nanorods Conjugated with Doxorubicin and cRGD for Combined Anticancer Drug Delivery and PET Imaging. Theranostics. 2012; 2: 757-68.

40. Lee SM, Park H, Choi JW, Park YN, Yun CO, Yoo KH. Multifunctional nanoparticles for targeted chemophotothermal treatment of cancer cells. Angew Chem Int Edit. 2011; 50: 7581-6.

41. Ramos J, Taylor D, Rege K. Gold Nanoparticle Mediated Photo-Chemotherapy. J Nanomed Nanotechol. 2012; 3: 8-9.

42. Wang S, Huang P, Nie L, Xing R, Liu D, Wang Z, et al. Single continuous wave laser induced photodynamic/plasmonic photothermal therapy using photosensitizer-functionalized gold nanostars. Adv Mater. 2013; 25: 3055-61.

43. Link S, Burda C, Nikoobakht B, El-Sayed MA. Laser-Induced Shape Changes of Colloidal Gold Nanorods Using Femtosecond and Nanosecond Laser Pulses. J Phys Chem B. 2000; 104: 6152-63.

44. Huang X, Peng X, Wang Y, Wang Y, Shin DM, El-Sayed MA, et al. A Reexamination of Active and Passive Tumor Targeting by Using Rod-Shaped Gold Nanocrystals and Covalently Conjugated Peptide Ligands. ACS Nano. 2010; 4: 5887-96. 